\title{
Análisis de resultados área de competencias ciudadanas en seis instituciones educativas de la ciudad de Medellín (Colombia)
}

\section{Results analysis citizen competences area in six educational institutions from Medellin city}

LUJAN, David A. ${ }^{1}$

LEMMEL-VÉLEZ, Karen ${ }^{2}$

RIOS, Rosalba ${ }^{3}$

MEJIA, Bernardo A. ${ }^{4}$

\begin{abstract}
Resumen
En este artículo se reflexiona sobre los resultados obtenidos en seis instituciones educativas de la ciudad de Medellín de los grados $3^{\circ}, 5^{\circ}, 7^{\circ}, 9^{\circ}$ y $11^{\circ}$ en cuanto a competencias ciudadanas se refiere. Se aplicaron pruebas de 20 ítems cada una con diferentes niveles de desempeño y complejidad. Se obtuvo como resultado la existencia de dificultades en relación a lo cognitivo y no cognitivo de las competencias ciudadanas.

Palabras clave: competencias ciudadanas, nivel de desempeño, nivel de dificultad

Abstract

This article reflects the results obtained in six educational institución in the city of Medellín in grades $3,5,7,9$ and 11 in terms of citizen competencies. Tests of 20 items were applied, each with different levels of performance and complexity. The result of these tests showed the existence of difficulties in relación to cognitive and non-cognitive citizenship skills (abilities)

key words: citizen competences, level of performance, level of difficulty
\end{abstract}

\section{Introducción}

La formación ciudadana se constituye hoy en una necesidad acuciante, formar en el respeto, la tolerancia, la participación, el reconocimiento del otro, el compromiso con lo público, la primacía de lo colectivo sobre lo particular, la comprensión de la realidad social, el reconocimiento de la estructura política del país y la capacidad de analizar, opinar y potenciar cambios, dejan de ser una posibilidad en el ámbito educativo para situarse en una imperante necesidad ante la realidad social del país.

\footnotetext{
${ }^{1}$ Licenciado en básica con énfasis en tecnología e informática, Especialista en Gerencia Informática, Magister en Educación. daluqui@yahoo.es 2 Ingeniera en Instrumentación y control, Magister en automatización y control industrial, PhD (c) en Educación. Departamento de eléctrica y afines. Institución Universitaria Pascual Bravo.karen.lemmel@pascualbravo.edu.co

${ }^{3}$ Ingeniera De Sistemas, Especialista en Auditoria de Sistemas, Especialista en Integración de Redes y tecnologías, Magister en Administración de Proyectos. Departamento de eléctrica y afines. Institución Universitaria Pascual Bravo ro.rios@pascualbravo.edu.co

${ }^{4}$ Licenciado en educación Física, Especialista En informática Educativa. Departamento de eléctrica y afines. Instituto Técnico industrial Pascual Bravo. bernardomejia@tecnicopascualbravo.edu.co
} 
La formación integral se entiende como aquella que potencia el desarrollo cognitivo, pero también el desarrollo humano por ende las experiencias de aprendizaje deben situar al sujeto educable en la posibilidad de crecer en ambos sentidos.

Las pruebas externas (Conocidas como pruebas Saber), cuyo objeto es reconocer las condiciones en las que se sucede el acto educativo, los avances, las herramientas de las que dispone el sujeto para enfrentar situaciones en las que ha de poner en juego sus aprendizajes, evidencian hoy algunas dificultades en relación a la formación ciudadana, situación que exige de los actores vinculados en el acontecimiento educativo, la generación de estrategias y acciones tendientes al fortalecimiento de la experiencias formativas sobre el componente humano, social, político, con el objeto no de mejorar unos resultados, sino las posibilidades de interacción social.

La realidad en la que se desenvuelve la sociedad colombiana se caracteriza por significativas particularidades y fenómenos que plantean una serie de retos y desafíos a los que deben enfrentarse todos aquellos organismos, entidades o instituciones encargadas de los procesos de socialización. La escuela como agente socializador por excelencia, enfrenta el desafío de responder a los fines de la educación bajo condiciones en muchos casos adversas y que son el resultado de las problemáticas y dificultades propias de la sociedad colombiana que confluyen en este escenario. Las marcadas diferencias socio-económicas, la presencia de grupos armados al margen de la ley que han legitimado su accionar, la coacción, terrorismo, desplazamiento, desempleo, pobreza, desintegración familiar, por mencionar algunos, han tenido injerencia en los procesos educativos exigiendo estrategias tendientes a disminuir los efectos nocivos que, a nivel de interacción, convivencia, postura frente a la vida, construcción de identidad e intersubjetividad se puedan dar. Estos resultados fueron obtenidos en el proyecto de Investigación "Un Transito a la educación Superior" que tiene como objetivo General : Analizar cómo desde un currículo alternativo se pueden articular los niveles de la educación formal y como Objetivos Específicos: se definieron los siguientes : 1.Analizar en las instituciones participantes del proyecto cómo es la dinámica del proceso de articulación desde la perspectiva currículo y desarrollo de competencias.2.Caracterizar cómo el diseño y el desarrollo del currículo del Instituto Técnico Industrial Pascual Bravo determinan la formación por competencias.3.Definir un currículo alternativo que permita la articulación entre las instituciones participantes y la educación superior, que proyecte un profesional formado en el SER para el CONOCER, el HACER y el CONVIVIR.

El proyecto fue concebido para un periodo de tres años, tiempo en el cual se ejecutaron tres fases: caracterización, implementación y evaluación, con una metodología desde el enfoque cualitativo y una perspectiva hermenéutica interpretativa, contando además con los siguientes cofinanciadores: La Agencia de Educación Superior de Medellín- SAPIENCIA; la Secretaría de Educación de Medellín , la Alianza Minero Energética y la Institución Universitaria Pascual Bravo, a través del grupo de investigación GIIEN adscrito a la facultad de ingeniería de la misma, responsable del proyecto.

Los resultados de la evaluación de estas competencias fueron entregados a la Secretaria de Educación del municipio de Medellín, y a los rectores de cada una de las Instituciones Educativas participantes en el proyecto. Con las que se tomaron decisiones referentes al proceso de articulación, media técnica y áreas evaluadas y la Secretaría de Educación, al equipo de educación técnica y tecnológica, fueron encontrados hallazgos muy importantes que ayudaron en el proceso de acompañamiento, asesoría y asistencia técnica que hace con las instituciones educativas oficiales de Medellín.

\subsection{Marco Teórico}

En la escuela tienen lugar no solo el desarrollo cognitivo, sino que entran en juego con marcada trascendencia las interacciones y relaciones interpersonales que dan cuenta de la realidad particular de quienes en este 
espacio convergen, las problemáticas sociales irrumpen con vigor a la escuela y se consolidan como un elemento que enmarca, delimita y precisa el accionar de esta a fin de establecer concomitancia entre la función de la escuela y la realidad del sujeto educable.

En grupos sociales fuertemente aquejados por problemática sociales, se evidencian disyuntivas a las que se hace imperativo responder. Las dificultades para la convivencia, la indiferencia ante las posibilidades de participación, el menosprecio por lo público, la supremacía de lo individual o particular sobre lo colectivo, entre otras situaciones, son el producto de esas realidades que confluyen en la escuela y que inciden en la postura social del sujeto.

Hoy en el ámbito educativo se exhibe una marcada fragilidad en lo relacionado al otro; su reconocimiento, respeto, aceptación, vinculación, se advierten como una problemática, como un factor adverso, aquello que debería serle natural a la escuela como escenario social se erige hoy como una situación necesaria de intervenir y atender en función de repotenciar la concepción de escuela como epicentro para el encuentro, el acercamiento, la fraternidad, es decir que la escuela potencie el surgimiento o preludio de un ser social y por ende un ser político, ético, democrático que vive su individualidad y particularidad en equilibrio y concordancia con lo colectivo, lo general, lo comunal, por tanto una escuela donde la agremiación y la asociación tenga no solo cabida sino protagonismo, es decir, hoy ante la realidad de nuestra sociedad, sus intereses y necesidades es imperativo que la educación sea concebida como acontecimiento, donde la visualización del otro y lo otro y de sí mismo en relación a los primeros, sea una constante, una necesidad más que una posibilidad, en otras palabras, la escuela habrá de generar estrategias pedagógicas que favorezcan el desarrollo, mejoramiento y de manera especial la institucionalización de habilidades para la coexistencia, formación entonces para el desarrollo de lo humano.

Resulta inquietante y desalentador reconocer en la sociedad y los estamentos que la conforman una tendencia a la homogenización, a la búsqueda de congruencias e igualaciones, a la estandarización, una imperiosa necesidad de presentar lo humano como un artificio, como algo superfluo que ha perdido validez y se diluye en el discurso económico y mercantilista de la globalización. Lo humano ocupa un lugar secundario, la escuela no escapa a la tendencia homogeneizante en la que se coartan las posibilidades de asumirse como individuos y sujetos plurales, diversos, mutilando de paso cualquier posibilidad de crecer en virtud de la experiencia interpersonal.

Reconocer la escuela como espacio para visibilizar al otro, implica también contemplar lo otro, contextualizar realidades, subjetividades, historias, devenires. Implica comprender realidades contextuales derivadas de trasformaciones y acontecimientos que determinan el rumbo de la humanidad, de la escuela, del sujeto. La otra cobra sentido y trascendencia como enlace, punto de partida o referente. En este sentido vale la pena mencionar a Nussbaum (2005) quien siguiendo esta línea plantea tres condiciones, competencias o habilidades para cultivar la humanidad, es decir aquella condición en la que el otro cuenta: “(...) habilidad para un examen crítico de uno mismo y de las propias tradiciones, que nos permita experimentar lo que, siguiendo a Sócrates, podríamos llamar 'vida examinada'” (p. 28); “(...) capacidad de verse a sí mismo no solo como ciudadanos pertenecientes a alguna región o grupo, sino también, y sobre todo, como seres humanos vinculados a los demás seres humanos por lazos de reconocimiento y mutua preocupación” (p. 29), “(...) puede llamarse imaginación narrativa. Esto significa la capacidad de pensar cómo sería estar en el lugar de otra persona, ser un lector inteligente de la historia de esa persona, y comprender las emociones, deseos y anhelos que alguien así pudiera experimentar" (p. 30).

El cometido de la educación es la formación integral, el desarrollo de las capacidades del sujeto, capacidades en relación a lo cognitivo, pero también en relación a lo afectivo, lo emocional, a pesar de la claridad frente a este elemento indisoluble la escuela continúa privilegiando la formación o desarrollo cognitivo lo que fractura 
o quebranta la mirada holística del ser. A este respeto en el informe De los (1996) es asume la educación a partir de cuatro pilares o fundamentos: "saber conocer, saber hacer, saber ser y saber convivir", esto es, un sujeto que conoce, comprende, propone, actúa, se conoce, busca ser mejor reconoce al otro y procura interacciones basadas en el respeto. Habrá de entenderse como paradójico, discordante, infructuoso desarrollar estrategias o acciones para lograr el cometido de "Colombia la mejor educada" si no se insertan de manera inmediata procesos de formación del ser, formación que trascienda el civismo, los buenos modales y los arquetipos sociales y se consolide como una formación para la fraternidad, la convivencia, la interacción en un mundo que conservando rasgos locales está cada vez más abierto al mundo, a la globalización, a aquello que pensadores y teóricos de la complejidad llamarían era planetaria. Se requieren así, mecanismos que traduzcan en realidades fácticas los principios axiológicos que sustentan la sociedad.

La educación tiene un cometido social, cometido al que al parecer no se está respondiendo de manera eficaz y eficiente, los sujetos que asisten a la escuela no evidencian el desarrollo de las habilidades o capacidades que requiere la sociedad, aquellos conocimientos y actitudes en función de lo social, de lo colectivo o lo público.

En los contextos educativos es común reconocer elementos que contradicen la idea de una escuela inclusiva e incluyente, tolerante, una escuela que como micro sociedad se desenvuelva en base en el respeto, la participación, el consenso, la primacía del bien colectivo, la defensa de los derechos y el acatamiento de los deberes. Esta situación no solo es latente en las realidades empíricas (aulas), los resultados de pruebas evidencias dificultades de carácter experiencial y conceptual, criticidad, análisis, reflexión y posibilidad para plantear o proponer alternativas de solución a situaciones cotidianas, es decir que se evidencian dificultades en relación a la formación de un ser social, de un ciudadano.

La concepción de ciudadano gana terreno en el escenario educativo del país con la constitución de 1991, donde se promueve la participación democrática, inclusión de minorías hasta entonces relegadas, libertad de culto y género, y una nueva y más holística postura frente al concepto de ciudadanía. Los planteamientos en el ámbito educativo se plantean el reto de una formación intelectual incluyendo elementos humanos, es decir, que la escuela potencie la formación de sujetos competentes, entendiendo la competencia como lo afirma Carlos Vasco como una "capacidad de movilizar conocimientos, valores, habilidades, actitudes, comprensiones y disposiciones, entre sí para facilitar el desempeño flexible, eficaz y consentido de una actividad o de cierto tipo de tareas en contextos relativamente nuevos y retadores.

Devolver la mirada sobre el ser humano es hoy más que un ejercicio derivado de la filantropía o la axiología, una imperante necesidad y un compromiso acuciante a fin de garantizar la coexistencia. Concebir la escuela como escenario para la formación integral, implica una mirada mesurada, pero a la vez vasta y profunda del ser como totalidad, como unidad, al respecto Morin (1999) cuando se pregunta por la educación del futuro plantea la pertinencia de comprender al hombre como un ser complejo precisado por su multi dimensionalidad al que fija como "biológico, psíquico, social, afectivo, racional".

Al respecto y reflexionando sobre el aporte de las competencias ciudadanas en la resolución de las problemáticas sociales Chaux (2005) plantea:

Hay un concepto que se utiliza en la teoría de sistemas cuando uno quiere transformar un sistema muy complejo, donde todo se relaciona con todo.

Lo que se hace es buscar los llamados nudos críticos, que cumplen con dos condiciones: por un lado, se tiene la posibilidad de afectarlo, por el otro si se logra cambiarlo, se transforma gran parte del sistema. La formación ciudadana es un nudo crítico, es decir, tenemos la posibilidad de afectar el sistema y hay herramientas claras para trabajar en eso (p.135). 
Hablar de formación ciudadana es abordar las complejas relaciones que se tejen en lo humano, pensar en formación ciudadana como apuesta por la integralidad del ser humano implica afrontar equilibrio entre lo individual y lo colectivo, entre lo deseable y lo posible, entre lo placentero y conveniente, entre el dar y el recibir, entre influir sobre un mundo que tiene gran influencia sobre el ser. Aquí las relaciones de interdependencia propias de los sistemas se hacen palpables, admirables e incluso explicables, aquí la autorregulación, el autocontrol como particularidad propia de los organismos vivos, se hace latente permeando al todo, es decir, el accionar como sujetos tiene una marcada incidencia en el contexto al que necesaria y por pluralidad de razones y relaciones se está anclado en un proceso de afectación no solo continuo sino bilateral en el que la membrecía cobra significancia y valor como patrón de adhesión

La formación ciudadana como insumo para la formación integral bajo una perspectiva compleja otorga un valor especial al otro, otro en torno al cual, en repetidas ocasiones, no se genera una reflexión de la que deriven acuerdos, donaciones solidarias, pensar en la posibilidad de entramados, conexiones, es pensar en el otro como prolongación del propio ser, como próximo, como cercano, incluso como intruso, pero jamás como lejano o remoto.

El ser humano formado en y para la humanidad ha de convertirse en la intencionalidad que diferencia a la escuela de otros escenarios formativos, parafraseando a Dewey la escuela es la partera de una democracia que se reconstruye en cada generación. Es aquí donde cobran relevancia la necesidad de que la escuela favorezca la racionalidad, pero también la sensibilidad, es aquí donde sujetos multidimensionales, actuando sobre y en entornos en dinámica de cambio podrán ajustar su accionar la su individualidad sin detrimento de la colectividad en proceso permanente de retroalimentación. Posibilitar pensamiento complejo, es decir racionalidad abierta y critica y reflexiva entonces, habrá de permitir la comprensión sobre las implicaciones de vivir en comunidad, es decir pensar lo particular dentro de un sistema más amplio en el que se generan interacciones determinantes no solo en la aparentemente simple relación entre unos y otros sino entre el otro y lo otro, de igual forma pensar la formación ciudadana desde un enfoque complejo implica la consideración de múltiples perspectivas que sustentan las posturas éticas y morales de los sujetos en los que la responsabilidad individual gana terreno en relación con el otro, una responsabilidad que deriva de la reflexión pero también de la pregunta.

Ubicados en espacios reales el proceso educativo se ha dirigido a llenar y completar en los sujetos que son implicados a dicha causa, un cúmulo de conocimientos que cada una de las áreas y/o ciencias tienen determinados en el currículo institucional homogenizando aprendizajes y comportamientos; dejando de un lado la importancia del otro como ser integral. Ese otro que complementa mi espacio, ese otro que llena mis expectativas, ese otro que tiene diferentes formas de pensar y sentir y por tener esas características llaman mi atención y me desestabilizan en cada momento compartido en el aula. Larrosa (2003) afirma:

La educación se concibe con algunos ideales públicos o personales como la igualdad, la democracia, el enriquecimiento de la vida cultural, el pleno desarrollo de las capacidades humanas, la racionalidad, la virtud, el diálogo, la comunidad, la autonomía y otros. Y esos ideales son los mismos con los que se construye también una representación moral de la sociedad, de la comunidad o de la persona humana. (p. 555)

"El ser humano es un ser participativo, un actor social, un sujeto histórico individual y colectivo, un ser constructor de relaciones sociales lo más igualitarias, justas, libres y fraternas que sea posible, dentro de determinadas condiciones históricas sociales" (Boff, 2002, p.31).

Retomando el concepto de competencia como saber hacer, se trata de ofrecer a los niños y niñas las herramientas necesarias para relacionarse con otros de una manera cada vez más comprensiva y justa y para que sean capaces de resolver problemas cotidianos. Las competencias ciudadanas permiten que cada persona 
contribuya a la convivencia pacífica, participe responsable y constructivamente en los procesos democráticos y respete y valore la pluralidad y las diferencias, tanto en su entorno cercano, como en su comunidad, en su país o en otros países. (pag.8)

Además, el Ministerio de Educación Nacional (2004), plantea una organización de dichas competencias en tres grupos:

- Convivencia y paz: se basan en la consideración de los demás y, especialmente, en la consideración de cada persona como ser humano.

- Pluralidad, identidad y valoración de las diferencias: parten del reconocimiento y el disfrute de la enorme diversidad humana y tienen, a la vez como límite, los derechos de los demás.

- Participación y responsabilidad democrática: se orientan hacia la toma de decisiones en diversos contextos, teniendo en cuenta que dichas decisiones deben respetar, tanto los derechos fundamentales de los individuos, como los acuerdos, las normas, las leyes y la Constitución que rigen la vida en comunidad" (p.12).

Propone además de estos grupos, unos tipos de competencias ciudadanas a saber:

- Los conocimientos se refieren a la información que los estudiantes deben saber y comprender acerca del ejercicio de la ciudadanía. Si bien esta información es importante, no es suficiente para el ejercicio de la ciudadanía y se necesitan las demás competencias.

- Las competencias cognitivas se refieren a la capacidad para realizar diversos procesos mentales, fundamentales en el ejercicio ciudadano. Por ejemplo, la habilidad para identificar las distintas consecuencias que podría tener una decisión, la capacidad para ver la misma situación desde el punto de vista de las personas involucradas, y las capacidades de reflexión y análisis crítico, entre otras.

- Las competencias emocionales son las habilidades necesarias para la identificación y respuesta constructiva ante las emociones propias y las de los demás. Por ejemplo, la capacidad para reconocer los propios sentimientos y tener empatía, es decir, sentir lo que otros sienten, por ejemplo, su dolor o su rabia.

- Las competencias comunicativas son aquellas habilidades necesarias para establecer un diálogo constructivo con las otras personas. Por ejemplo, la capacidad para escuchar atentamente los argumentos ajenos y para comprenderlos, a pesar de no compartirlos. O la capacidad para poder expresar asertivamente, es decir, con claridad, firmeza y sin agresión, los propios puntos de vista.

- Las competencias integradoras articulan, en la acción misma, todas las demás. Por ejemplo, la capacidad para manejar conflictos pacífica y constructivamente, que es una competencia integradora, requiere de ciertos conocimientos sobre las dinámicas de los conflictos, de algunas capacidades cognitivas como la habilidad para generar ideas y opciones creativas ante una situación de conflicto, de competencias emocionales como la autorregulación de la rabia, y de ciertas competencias comunicativas como la capacidad para transmitir asertivamente los propios intereses. (p.12-13)

\section{Metodología}

Las competencias ciudadanas adquieren relevancia en la escuela como estrategia tendiente a suplir ese vacío en relación al ser, un ser social, capaza de pensarse como miembro de una colectividad que espera de él sus mejores contribuciones, donaciones y aportes. El Ministerio de Educación Nacional (2006) define las competencias como "Conocimientos, habilidades, actitudes, comprensiones y disposiciones cognitivas, socio afectivas y psicomotoras apropiadamente relacionadas entre sí, para facilitar el desempeño flexible, eficaz y con sentido de una actividad en contextos relativamente nuevos y retadores" (p. 49). Con La Guía N6 Estándares Básicos de Competencias Ciudadanas el Ministerio de Educación Colombiano plantea una 
herramienta o carta de navegación a fin de tener claridad frente a la pertinencia de las competencias ciudadanas como un componente clave en el proceso formativo y esboza sus principales acepciones frente a las mismas, estableciendo claridad frente a definición y vinculación en el proceso educativo. En este documento el MEN (2003) determina las competencias ciudadanas como:

El conjunto de conocimientos y de habilidades cognitivas, emocionales y comunicativas que, articulados entre sí, hacen posible que el ciudadano actúe de manera constructiva en la sociedad democrática.

En base a lo propuesto por el ministerio respecto a la definición, organización y tipos de competencias en lo que a competencias ciudadanas respecta se generaron 20 ítems (preguntas) por grado a evaluar $\left(3^{\circ}, 5^{\circ}, 7^{\circ}, 9^{\circ} \mathrm{y}\right.$ $11^{\circ}$ ), que hacen parte de una Prueba aplicada en seis Instituciones Educativas del municipio de Medellín, Colombia, estas preguntas se clasifican en Niveles de Dificultad - Complejidad, que corresponden a la siguiente clasificación.

\section{Nivel I - Complejidad Baja Ítem Simple}

El estudiante requiere un manejo y conocimiento solo de conceptos básicos del área.

\section{Nivel II - Complejidad Media Ítem Mediano - Estándar}

El estudiante requiere de un manejo y conocimiento de conceptos fundamentales del área evaluada, sin requerir de conocimientos profundos ni teorías.

\section{Nivel III - Complejidad Alta Ítem Complejo y Difícil}

Requiere dominio del área por parte del estudiante que le permita una comprensión más profunda y analítica de la temática evaluada, con algunos conocimientos teóricos.

\section{Nivel de Desempeño Avanzado}

Evidencia por parte del estudiante un dominio del área de manera que le permite una comprensión de la temática evaluada.

\section{Nivel de Desempeño Satisfactorio}

El estudiante ha logrado asimilar conceptos fundamentales del área evaluada, por lo tanto, comprende en gran medida la temática evaluada.

\section{Nivel de Desempeño Mínimo}

El estudiante solo alcanza resultados básicos en el área evaluada, necesitando una nivelación que le permita una mejor asimilación de los conceptos básicos del área.

\section{Nivel de Desempeño Insuficiente}

El estudiante no ha logrado asimilar la temática evaluada, por lo tanto, desconoce conceptos fundamentales de esta y se muestra inseguro a la hora de responder.

Las pruebas se aplicaron a 80 estudiantes escogidos aleatoriamente de los grados $3^{\circ}, 5^{\circ}, 7^{\circ} 9^{\circ}(20$ de cada grado), además de 20 estudiantes de grado $11^{\circ}$.

\section{Resultados}

En las seis instituciones participantes del proyecto los resultados de dichas pruebas, al igual que las demás pruebas externas que se han aplicado en el componente de competencias ciudadanas, dejan en evidencia la existencia de dificultades en relación a lo cognitivo y lo no cognitivo, a las competencias emocionales, integradoras, comunicativas y cognitivas frente a los tres grupos de competencias ciudadanas. Situación que llama la atención en tanto la relevancia de este componente en la formación integral. 
Cabe la pregunta por la relevancia otorgada a los procesos de humanización, sobre las experiencias democráticas y ciudadanas vinculadas a la escuela, sobre los agentes socializadores, y demás elementos que pueden incidir en el éxito o fracaso de los procesos formativos en este componente. Reflexionar sobre las causas u orígenes de los bajos resultados en una prueba que da cuenta de la calidad de ciudadanos que se están formando debería ser el eje en torno al cual se establezcan planes en los que se diseñen e institucionalicen estrategia en las que se contemple: transversalición real del currículo, concepción holística del proceso formativo, praxeología como base del aprendizaje, entre otras tendientes a fortalecer el proceso formativo en todas las áreas del conocimiento. Entablar por fin una mirada sistémica del proceso educativo, donde no haya prioridades, donde no se deje nada al azar, donde lo multidisciplinar, interdisciplinar, pero ante todo lo multidimensional cobren vida en los escenarios educativos en cualquier nivel o ciclo formativo.

Pensar en la sociedad actual, en el hombre que reclama esa sociedad, en las acciones que habrá de liderar la escuela para atender a esa demanda, entre otros cuestionamientos de orden epistemológico implican rupturas de paradigmas, miradas complementarias, holísticas y abarcadoras, racionalidades abiertas, críticas y complejas en procura de establecer las conexiones e interrelaciones que al interior de la sociedad se suscitan y que la configuran como un sistema.

Explícitamente los resultados alcanzados por grado y niveles de desempeño de las seis instituciones se muestran en las figuras 1 a la 5.

Figura 1

Resultados Competencias ciudadanas

grado $3^{\circ}$ por niveles de desempeño

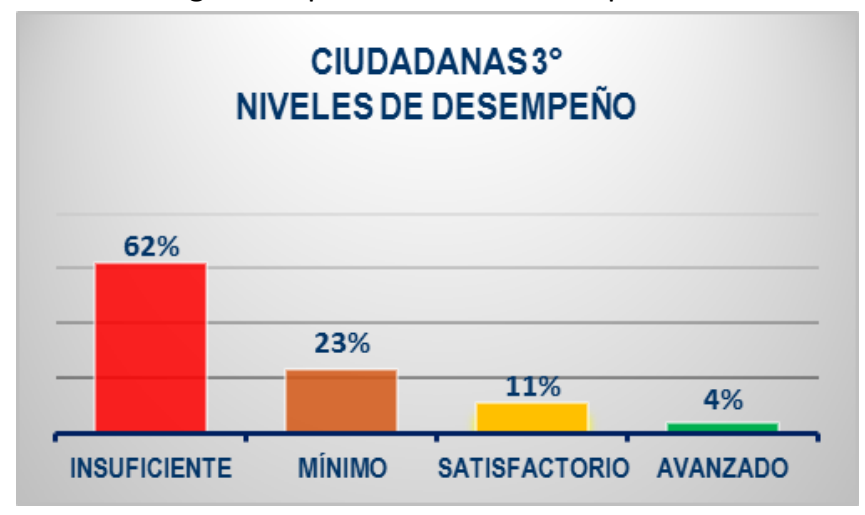

Figura 2

Resultados Competencias ciudadanas

grado $5^{\circ}$ por niveles de desempeño

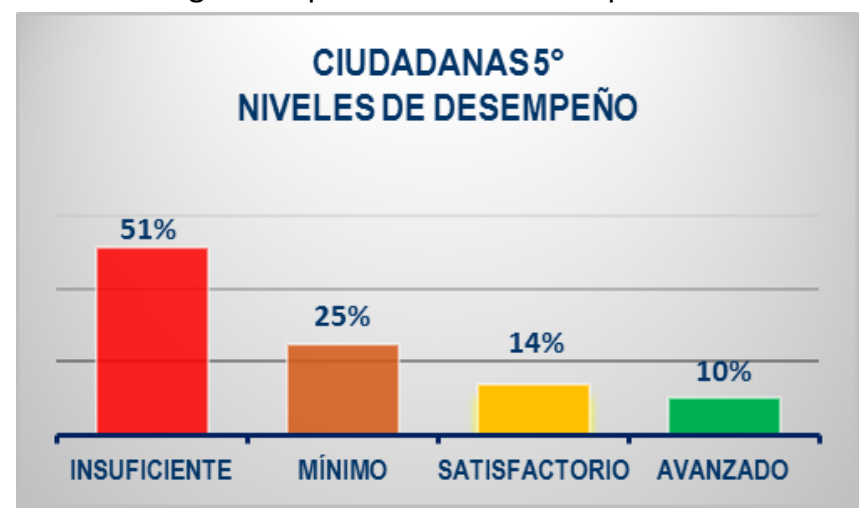


Figura 3

Resultados Competencias ciudadanas

grado $7^{\circ}$ por niveles de desempeño

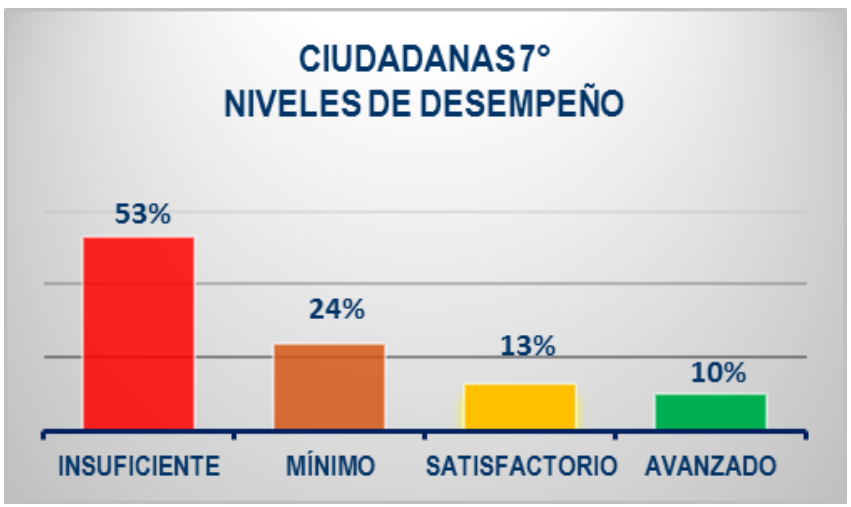

Figura 4

Resultados Competencias ciudadanas

grado $9^{\circ}$ por niveles de desempeño

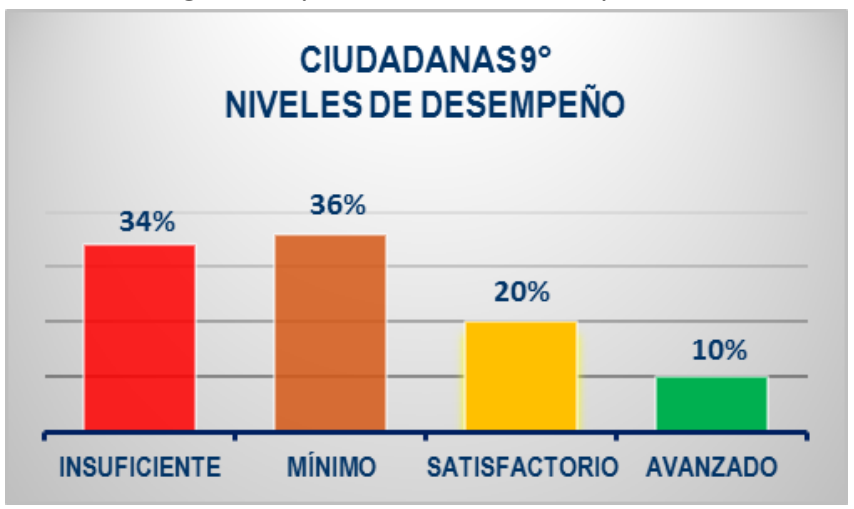

Figura 5

Resultados Competencias ciudadanas

grado $11^{\circ}$ por niveles de desempeño

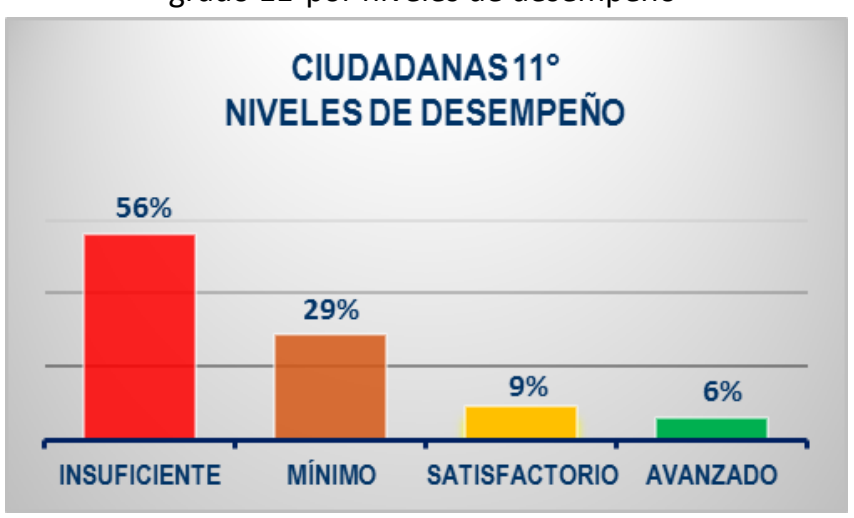

Los resultados alcanzados por grado y niveles de dificultad de las seis instituciones se muestran en las figuras 6 a la 10. 
Figura 6

Resultados Competencias ciudadanas grado $3^{\circ}$ por niveles de dificultad

PORCENTAJE DE RESPUESTAS CORRECTAS SEGÚN NIVEL DE DIFICULTAD CIUDADANAS GRADO $3^{\circ}$

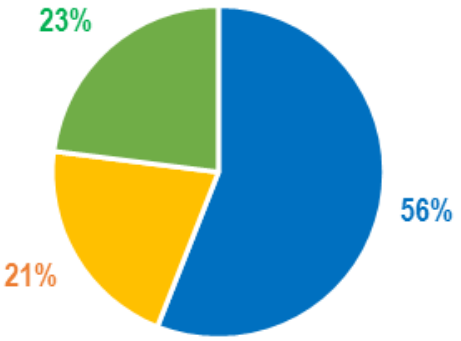

Figura 7

Resultados Competencias ciudadanas grado $5^{\circ}$ por niveles de dificultad

PORCENTAJE DE RESPUESTAS CORRECTAS SEGÚN NIVEL DE DIFICULTAD CIUDADANAS GRADO $5^{\circ}$

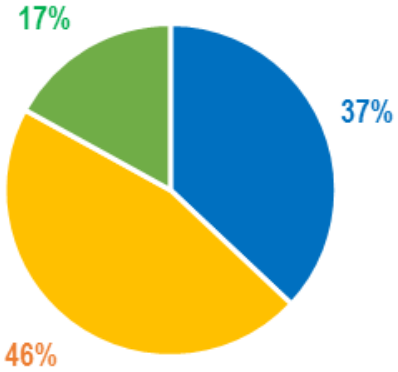

Figura 8

Resultados Competencias ciudadanas grado $7^{\circ}$ por niveles de dificultad

\section{PORCENTAJE DE RESPUESTAS CORRECTAS} SEGÚN NIVEL DE DIFICULTAD CIUDADANAS GRADO $7^{\circ}$

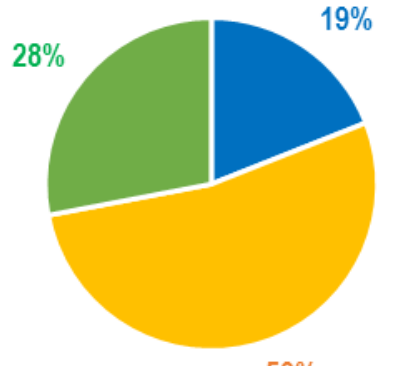


Figura 9

Resultados Competencias ciudadanas grado $9^{\circ}$ por niveles de dificultad

PORCENTAJE DE RESPUESTAS CORRECTAS SEGÚN NIVEL DE DIFICULTAD CIUDADANAS GRADO $9^{\circ}$

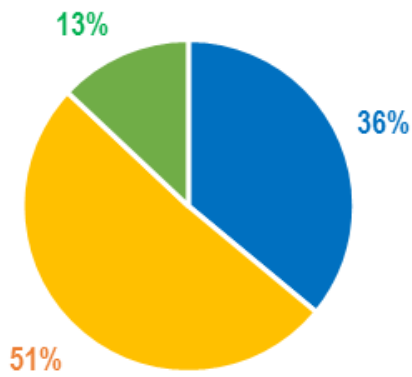

Figura 10

Resultados Competencias ciudadanas grado $11^{\circ}$ por niveles de dificultad

\section{PORCENTAJE DE RESPUESTAS CORRECTAS}

SEGÚN NIVEL DE DIFICULTAD CIUDADANAS $11^{\circ}$

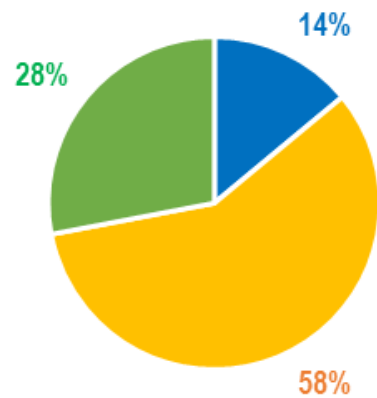

\section{Conclusiones}

La inquietud por humanizar la educación, por otorgar al otro un papel protagónico, por potenciar la responsabilidad, la justicia, el respeto, la conciencia de especie o grupo, el valor por lo público, la legalidad, el empoderamiento, entre otros no obedece a tendencias, discursos humanistas, intensiones personales o posibilidades de la escuela, está debe constituirse en un complemento esencial, imposible de obviar en tanto responde no solo a la condición de integralidad del sujeto sino a las demandas y necesidades de la sociedad actual.

Comprender que en la sociedad posmoderna la educación es valorada bajo indicativos de calidad y productividad ha menguado e incluso aniquilado en algunos momentos la concepción de educación como posibilidad de humanización, humanización no solo necesaria sino ineludible en momentos de segregación, aniquilación y violencia

En una sociedad con grandes problemáticas de todo orden, constantemente y desde diversas perspectivas se gestan propuestas que buscan contrarrestarlos, es así como las competencias ciudadanas buscan proveer las herramientas que permitan y posibiliten bajo parámetros de justicia, solidaridad y confraternidad, la 
concomitancia, donde los debates, disentimientos y confrontaciones no se eviten, sino que se enfrenten y solucionen sin recurrir a la violencia, la anulación o la negación. Se hacen necesarias posturas abarcadoras que se alejen de las tradicionales formas reduccionistas de concebir el mundo y las dificultades que en él se presentan. Pensar en la sociedad como sistema potencia y permite la mirada de un mundo en el que existen muchos mundos.

Nuestra sociedad concibe la idea de que el aula es el lugar donde se desarrolla una práctica educativa que lleva exclusivamente el proceso de enseñar y aprender conceptos o conocimientos precisos de una o varias disciplinas, perdiendo de vista el invitar a repensar el saber, la formación, el aprendizaje, la enseñanza, la democracia, la formación política, ética y ciudadana como acontecimiento en los que se tejen alternativas, dudas, necesidades, oportunidades, experiencias de cada uno de los participantes del proceso educativo y social.

Educar implica miradas y acepciones de que la cultura se conserva y se reproduce. Es reconocer que el que aprende captura, se transforma y se desarrolla como persona, abriéndose, desenvolviéndose en un despliegue de humanidad; de ahí que la educación potencie todas las habilidades del educando, de la familia y del mismo docente.

Las competencias ciudadanas buscan que las instituciones educativas se conviertan en entes articuladores, potenciadores, generadores de experiencias tendientes a fortalecer los conocimientos respecto a la ciudadanía. Con el ánimo de reconocer como se están desarrollando las competencias ciudadanas en las instituciones educativas del país, la eficacia de las estrategias utilizadas en su abordaje, las capacidades de los estudiantes de poner en práctica sus saberes y evidenciar sus avances, es decir, medir los desempeños de los estudiantes respecto a la formación ciudadana, el ICFES va venido realizando las pruebas Saber, que en este componente evalúa elementos cognitivos como: Conocimientos sobre la Constitución Política de Colombia, Valoración de argumentos, Multi perspectivismo y Pensamiento sistémico, también evalúa elementos no cognitivos: competencias emocionales centrales para la ciudadanía: empatía y el manejo de las emociones. Estas Arcudi, L., cuenta del ejercicio ciudadano.

\section{Referencias bibliográficas}

Arcudi, L., Bermúdez, A., Borrero, C., Bravo, A., Brion, Steve., Castilleja, P., et al. (2005). Comprensiones sobre ciudadanía. Bogotá: Transversales Magisterio.

Boff, L. (2002). El cuidado esencial. Ética de lo humano compasión de la Tierra. Madrid, Editorial Trotta.

Ciurana, E. (2008). Algunas reflexiones en torno a la comprensión compleja de la educación. Revista de investigación Universidad Católica de Manizales. 08(11), 14-21

Chaux, E. y Ruiz, A. (2005).La formación de competencias ciudadanas. Bogotá: 21 Editorial.

Constitución Política de Colombia. 1991

Delors, J. (1996) Los cuatro pilares de la educación. La educación encierra un tesoro. Informe a la UNESCO de la Comisión internacional sobre la educación para el siglo XXI, Madrid, España: Santillana/UNESCO. pp. 91103

Larrosa, J. (2003). La experiencia de la lectura. Estudios sobre literatura y formación. México: Fondo de Cultura Económica

Mieles, M. D. \& Acosta, A. (2012). Calidad de vida y derechos de la infancia: un desafío presente. Revista Latinoamericana de Ciencias Sociales, Niñez y Juventud, 10 (1), pp. 205-217. 
Mieles Barrera, María Dilia; Alvarado Salgado, Sara Victoria. (2012). Ciudadanías y competencias ciudadanas. Estudios Políticos, Enero-Junio, 53-75.

Ministerio de Educacion Nacional. (2003). Portal Colombia aprende. Recuperado el 22 de Febrero de 2015, de Portal Colombia aprende: http://www.colombiaaprende.edu.co/html/docentes/1596/article-58541.html

Ministerio de Educación Nacional. (2004). Formar para la ciudadanía isí es posible! Series Guías № 6. Estándares Básicos de Competencias Ciudadanas. Bogotá: Editorial IPSA.

Moran, E. (1999). Los siete saberes necesarios para la educación del futuro. UNESCO

Moran, E., Ciruana, E., Mota, R. (2002). Educar en la era planetaria. Barcelona: Gedisa

Nussbaum, M. (2005). El cultivo de la humanidad. Barcelona: Ediciones Paidós Ibérica, S. A

Ruíz, L. (2007). Formación integral: desarrollo integral, emocional, social y ético de los estudiantes. Revista Universidad de Sonora, vol. 19, p. 11-13

Stiglitz, J. (2006). Cómo hacer que funcione la globalización. Madrid, España: Taurus.

Toro, C., Ospina, L. y Vélez, L. (2014). “Formación para la ciudadanía, una mirada a la norma desde el manual de convivencia: un estudio de caso de la institución educativa San Pablo de la comuna 1 de la ciudad de Medellín". (Tesis de maestría). Universidad de Antioquia, Medellín, Colombia.

Vasco, C. (1999). Algunas reflexiones sobre la pedagogía y la didáctica. En: pedagogía, discurso y poder. CORPRODIC: Bogotá.

Vasco, C. y Delgado, R. (2007). Interrogantes en torno a la formación de las competencias ciudadanas y la construcción de lo público. En Yory, C. (ed) Espacio Público y formación de ciudadanía. Bogotá: Editorial Pontificia Universidad Javeriana

Esta obra está bajo una Licencia Creative Commons Attribución-NoCommercial 4.0 International

\section{(cc) EY-NC}

